\title{
Localized Sphingolipid Signaling at Presynaptic Terminals Is Regulated by Calcium Influx and Promotes Recruitment of Priming Factors
}

\author{
Jason P. Chan and Derek Sieburth \\ Zilkha Neurogenetic Institute, University of Southern California, Keck School of Medicine, Los Angeles, California 90033
}

Activity-dependent changes in presynaptic function represent a critical mechanism by which synaptic strength is controlled. However, how changes in synaptic activity couple to presynaptic components to control synaptic vesicle release and recycling are poorly understood. Sphingosine kinase (SphK) is a sphingolipid metabolic enzyme whose activity-dependent recruitment to membrane regions within presynaptic terminals promotes neurotransmitter release. Here, we show that synaptic recruitment of SPHK-1, the SphK ortholog in Caenorhabditis elegans, is mediated by presynaptic calcium influx. Quantitative fluorescence imaging of live presynaptic terminals reveals that blocking presynaptic calcium influx reduces synaptic SPHK-1 abundance whereas increasing calcium influx increases SPHK-1 synaptic abundance. CALM-1, the calcium and integrin binding protein ortholog, colocalizes with SPHK-1 at release sites and regulates muscarinic-mediated synaptic SPHK-1 recruitment. We identify two additional sphingolipid metabolic enzymes that are concentrated at presynaptic terminals, and mutants lacking one of these, HYL-1/ceramide synthase, have defects in synaptic transmission and in synaptic vesicle cycling. Finally, we show that SPHK-1 activity is required for the recruitment of the priming protein UNC13/Munc13 to presynaptic terminals following activation by muscarinic signaling. These findings suggest that calcium-dependent regulation of local S1P metabolism at synapses may be an important mechanism by which synaptic vesicle priming factors are recruited to release sites to promote synaptic transmission.

\section{Introduction}

Regulation of neurotransmitter release allows synapses to alter their strength in response to changes in activity and environmental conditions. Presynaptic calcium influx plays a critical role in mediating these responses by regulating synaptic vesicle release and recycling, and by activating second messengers involved in synaptic plasticity (Catterall and Few, 2008). However, the molecular mechanisms by which calcium regulates presynaptic signaling pathways to modulate neurotransmitter release are poorly understood.

Sphingosine kinase (SphK) and its metabolites, the bioactive lipids sphingosine (SPH) and sphingosine-1-phosphate (S1P), regulate diverse cellular functions in the nervous system including cell survival, differentiation, and neurotransmitter secretion (Okada et al., 2009). In particular, S1P facilitates neuronal firing and neurotransmitter release from neurons and neuroendocrine

\footnotetext{
Received June 12, 2012; revised Sept. 24, 2012; accepted 0ct. 23, 2012.

Author contributions: J.P.C. and D.S. designed research; J.P.C. and D.S. performed research; J.P.C. and D.S. analyzed data; J.P.C. and D.S. wrote the paper.

This work was supported by grants from the American Heart Association and the National Institutes of Health to D.S. (NS071085). We thank the Caenorhabditis Genetic center and the National BioResource Project for strains used in this study. We thank Q. Cn'ng, T. Staab, H. Wang, and M. Olahova for critical reading of this manuscript; S. Gorda, C. Gillian, J. Delcarson, and T. Staab for technical assistance; and A. van der Bliek for the mitochondrial markers. The authors declare no competing financial interests.

Correspondence should be addressed to Derek Sieburth, Zilkha Neurogenetic Institute, University of Southern California, 1501 San Pablo Street, Los Angeles, California 90033. E-mail: sieburth@usc.edu.

DOI:10.1523/JNEUROSCI.2808-12.2012

Copyright $\odot 2012$ the authors $\quad 0270-6474 / 12 / 3217909-12 \$ 15.00 / 0$
}

cells and SPH drives synaptic vesicle exocytosis by facilitating SNARE protein assembly (Darios et al., 2009; Okada et al., 2009). Genetic ablation or knockdown of SphK activity results in reductions in electrically evoked neurotransmitter release, defects in long-term potentiation, and accumulation of synaptic vesicles at presynaptic terminals (Kajimoto et al., 2007; Kanno et al., 2010; Chan et al., 2012). SphK rapidly translocates to presynaptic terminals in response to neuronal activity and to presynaptic heterotrimeric G-protein signaling. Depolarization or treatment with muscarinic agonists results in rapid increase in synaptic translocation of SphK in hippocampal cultures or Caenorhabditis elegans motor neurons, respectively. On the other hand, hyperpolarization or reductions in muscarinic signaling decrease in SphK synaptic abundance (Kajimoto et al., 2007; Chan et al., 2012). Because studies in diverse cell types show that a critical step in regulating SphK activity is to promote its translocation to cellular membranes from cytosolic pools, identifying mechanisms underlying its translocation to synapses will be important for understanding the regulation of presynaptic plasticity.

Calcium has been implicated in regulating SphK translocation to cellular membranes. In neuroblastoma cells, calmodulin (CaM) inhibitors block translocation of SphK to plasma membranes (Young et al., 2003). In HEK cells, calcium mediates the interaction between SphK and calcium and integrin binding protein (CIB) and CIB knockdown impedes agonist-dependent SphK membrane translocation and S1P production (Jarman et al., 2010; Pitson, 2011). However, whether calcium mobilization mediates SphK translocation to synaptic membranes, how neu- 
ronal activity and heterotrimeric G-protein signaling converge at synapses to regulate SphK, and how synaptic SphK, in turn, promotes neurotransmitter release are not known.

Here, we show that presynaptic calcium influx regulates the recruitment of SPHK-1 to presynaptic membranes in C. elegans motor neurons. Muscarinic-mediated recruitment of SPHK-1 to synapses requires presynaptic calcium influx as well as the interaction of SPHK-1 with the C. elegans CIB ortholog, CALM-1. Furthermore, HYL-1/ceramide synthase, which also localizes to presynaptic terminals, antagonizes the effects of $s p h k-1$ on synaptic transmission. Finally, sphk-1 activity mediates the effects of muscarinic signaling by recruiting the synaptic vesicle priming protein UNC-13/Munc13 to synapses. Together, these findings suggest that local sphingolipid metabolism at synapses may be a previously unrecognized mechanism by which presynaptic calcium regulates presynaptic function.

\section{Materials and Methods}

C. elegans strains. All experiments were performed on young adult hermaphrodites. Strains sphk-1 (ok1097) and hyl-1(gk203) were provided by the Caenorhabditis Genetics Center, which is funded by the National Institutes of Health-National Center for Research Resources. Strain calm-1(tm1353) was provided by National BioResource Project (Japan). The wild-type reference strain was N2 Bristol. sphk-1 (ok1097) was outcrossed 10 times and used for all experiments. All other strains used were outcrossed more than four times. In addition, the following strains were used: egl-19(ad1006lf), egl19(n2368gf), egl-30(js126gf) [strain information on www.wormbase.org], unc-2(Ij1), unc-13(s69), unc-68(r1162), unc-104(e1265), nuIs46[myo-2GFP, Punc-129-unc13S-GFP], nuIs152 [ttx-3-mRFP, Punc-129-GFP-snb-1], nuIs197[myo-2-GFP, Punc-129-sphk-1-GFP]. All integrated transgenes were outcrossed 10 times.

Molecular biology. The promoter fragment of sphk-1 was amplified by PCR from mixed-stage genomic DNA. sphk-1 mutations were made by standard molecular cloning techniques or by Quikchange PCR (Stratagene). sphk-1 $(\Delta \mathrm{CaM})$ contains F217A, I272Q substitutions. Primers used for molecular cloning are available upon request. The following plasmids were made by standard molecular biology techniques (plasmid name[promotergene-marker]): pDS221[Punc-129-sphk-1-gfp], pDS287[Punc-129-hyl-1gfp], pDS297[Punc-129-sphk-1(NT1)-gfp], pDS300[Psphk-1-gfp-nls], pDS301[Punc-129-sphk-1-mCherry], pDS302[Pmyo-3-sphk-1-gfp], pDS303 [Psphk-1-sphk-1-gfp], pDS306[Punc-129-sphk-1( $\Delta \mathrm{CaM})$-gfp], pDS329[Pmyo3-OMM(inverted)-mCherry], pDS338[Punc-129-tag-38-gfp], pDS386[Punc129-sphk-1(NT3)-gfp], pDS387[Punc-129-sphk-1(NT2)-gfp], pDS388 [Punc-129-sphk-1(CT1)- gfp], pDS389[Punc-129-calm-1-mCherry], pDS391 [Punc-129-snn-1-mCherry], pDS394[Pcalm-1-gfp].

Transgenic lines. Transgenic strains were generated by injecting N2 or nuIs197 animals with expression constructs $(10-25 \mathrm{ng} / \mu \mathrm{l})$ and the coinjection marker [KP\#708 (Pttx-3-rfp, $40 \mathrm{ng} / \mu \mathrm{l})$ or KP\#1106 (Pmyo-2-gfp 10 $\mathrm{ng} / \mu \mathrm{l})]$. Microinjection was performed using standard techniques as previously described (Mello et al., 1991). At least three lines for each transgene were examined and a single, representative transgene was used for the experiments. The following transgenic lines were made (name[plasmid]): vjEx38[pDS390], vjEx39[pDS300], vjEx84[pDS329], vjEx98[pDS391], vjEx133[pDS302], vjEx337[pDS306], vjEx651[pDS297], vjEx652[pDS386], vjEx653[pDS387], vjEx654[pDS388], vjEx655[pDS389], vjEx656[pDS287], vjEx657[pDS338], vjEx711[pDS394].

Behavioral analysis of locomotion activity and sensitivity to aldicarb. For analysis of sensitivity to inhibitors of acetylcholinesterase (AChE), paralysis of adult worms was scored every $10 \mathrm{~min}$, starting at $40 \mathrm{~min}$ using 1.0 mM aldicarb (Chem Services). For each experiment, three plates of 20 worms per genotype were placed on NGM plates supplemented with aldicarb, and the number of worms paralyzed on each plate was counted to extract the percentage paralyzed at each time point per genotype. The percentages were averaged at each time point per genotype and plotted graphically. For each experiment, the genotype was blind to the scorer and the analysis was repeated at least two times. For experiments using arecoline, worms were pretreated with plates containing either control
M9 or arecoline (15 mm in M9) for $2 \mathrm{~h}$, which was determined to be the minimum concentration that produced maximal aldicarb hypersensitivity, to minimize nonspecific effects of the drug. For experiments using EGTA, worms were pretreated in drops of either EGTA ( $50 \mathrm{~mm}, \mathrm{pH} 7.2)$ or buffered water, $\mathrm{pH} 7.2$, for $2 \mathrm{~h}$ at room temperature. Concentrations were determined by experiments previously successful in other studies (Xu et al., 2001; O'Halloran et al., 2009; Earls et al., 2010). Statistical differences were determined at each time point by Student's $t$ tests.

Microscopy and analysis. Fluorescence microscopy experiments were performed as previously described (Ch'ng et al., 2008). Briefly, adult worms were paralyzed using 2,3-butanedione monoxime $(30 \mu \mathrm{g} / \mu \mathrm{l}$, Sigma) and mounted on $2 \%$ agarose pads for imaging. Images of synapses were captured from dorsal axons of DA/DB class motor neurons near the posterior gonadal bend of the worm. Quantification spanned $>100$ synapses for all experiments and specific $n$ values are shown in Table 1). For all fluorescence microscopy experiments, images were captured with a Nikon eclipse $90 i$ microscope equipped with a Nikon PlanApo $100 \times$ objective $(\mathrm{NA}=1.4)$ and a Photometrics Coolsnap ES ${ }^{2}$ camera. MetaMorph 7.0 software (Universal Imaging/Molecular Devices) was used to capture serial image stacks, and the maximum intensity projection was used for analysis of the dorsal cords. Line scans of the maximum intensity projection image were also recorded using MetaMorph. The fluorescence intensity values were then quantified using Puncta 6.0 software written with Igor Pro (Wavemetrics), as previously described (Dittman and Kaplan, 2006; Ch'ng et al., 2008). For all experiments, fluorescence values were normalized to the values of $0.5 \mu \mathrm{m}$ FluoSphere beads (Invitrogen) captured during each imaging session. This was performed to provide a standard for comparing absolute fluorescence levels between animals from different sessions.

We found that the conditions used for imaging showed that SPHK-1GFP were predominantly localized to synaptic puncta, as determined previously (Chan et al., 2012). There was little or no difference between their interpunctal fluorescence (in axons) and the autofluorescence observed at C. elegans axons. Therefore, we excluded the interpunctal fluorescence in our analysis for these markers (except for Fig. $4 c$ and Table 1, in which mutations in SPHK-1-GFP caused increased interpunctal fluorescence).

Statistical analysis. A Student's $t$ test was used to determine significance when comparing the fluorescence of SPHK-1-GFP in different conditions (significance was set at $p<0.05$ ). Propagation of SEM was performed for all comparisons. Statistical analyses for all experimental comparisons are presented in Table 1.

\section{Results}

\section{SphK expression and localization in C. elegans}

Mammalian SphKs are broadly expressed in many tissues including the brain, and they associate with diverse cellular membranes, including the nuclear envelope, plasma membrane, endoplasmic reticulum, and mitochondria (Siow et al., 2011). We previously showed that $C$. elegans SphK, sphk-1, is expressed in motor neurons of the ventral nerve cord, where it localizes to presynaptic membranes near synaptic vesicle release sites (Chan et al., 2012). Here, we determined the expression and localization pattern of SPHK-1 in other neuronal compartments and in other tissues. Transgenic animals expressing green fluorescent protein (GFP) under the control of a fully rescuing sphk-1 promoter fragment displayed fluorescence in multiple tissues, including the hypodermis (skin), muscles, the intestine, excretory canal cells, and some neurons in the head and tail (Fig. 1a).

In DA/DB class cholinergic motor neurons, functional GFPtagged SPHK-1 fusion proteins (SPHK-1-GFP) were detected in extranuclear regions in cell bodies (Fig. 4b), likely corresponding to Golgi (Sumakovic et al., 2009), occasionally in puncta in dendrites (data not shown), and in puncta at en passant presynaptic terminals (Chan et al., 2012). Colocalization studies revealed that SPHK-1-GFP was closely apposed to but not colocalized with the synaptic vesicle (SV) and actin-associated protein SNN-1/synap- 
Table 1. Quantification of fluorescent synaptic protein distribution

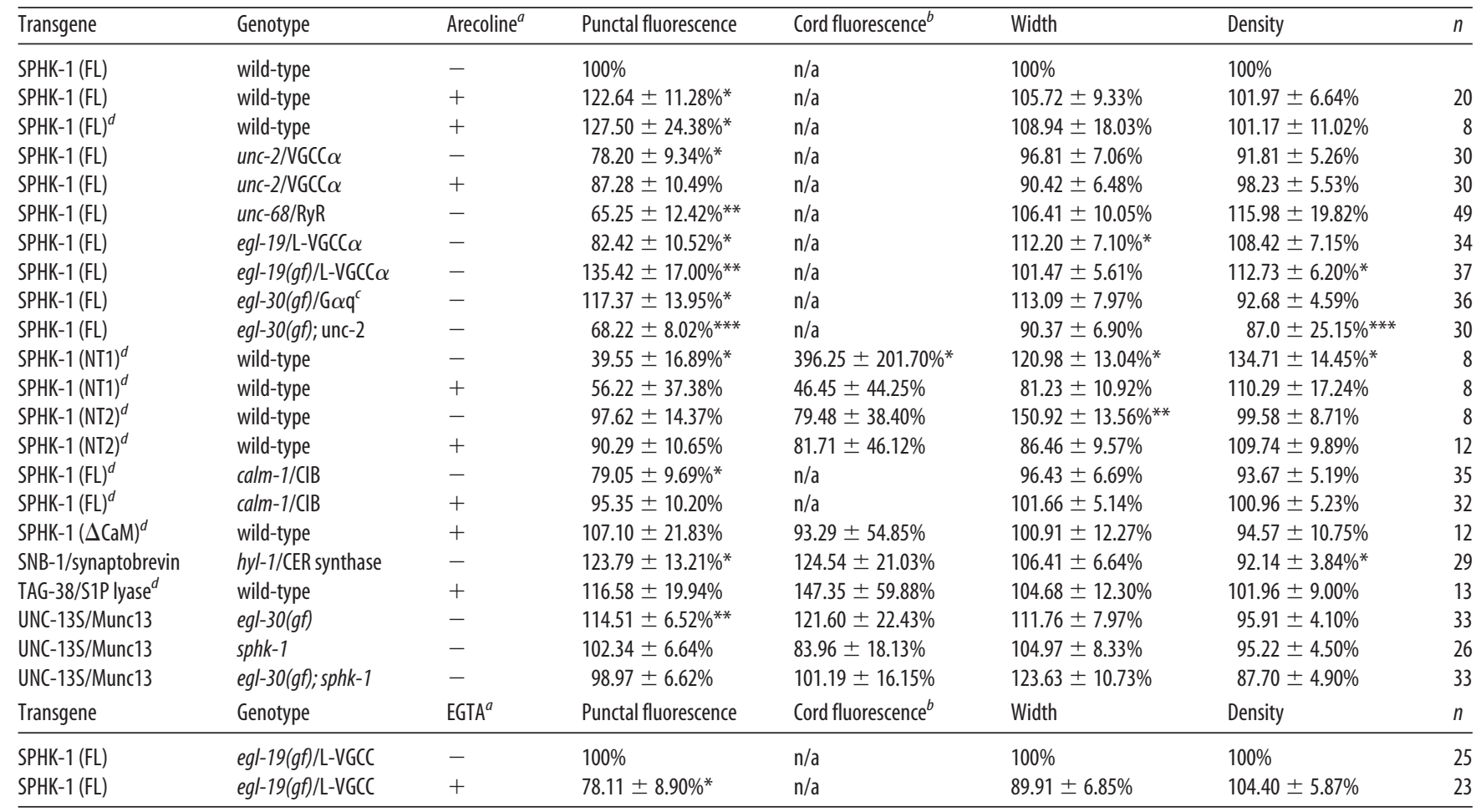

GFP-tagged transgenes were analyzed by fluorescence microscopy. Data is presented as comparisons of fluorescence values of mutants compared to wild-type (WT). ${ }^{a}$ Values for drug-treatment experiments are expressed as fluorescence values of drug-treated/control-treated animals. Arecoline and EGTA treatment was for $2 \mathrm{~h}$ and control treatment was $\mathrm{M} 9$ and water, respectively. SPHK-1-GFP cord fluorescence was omitted because it was not reliably detected above background, except for SPHK-1 truncation variants. Data was also used in (Chan et al., 2012). ${ }^{d}$ Transgene is an extrachromasomal array. n/a, not applicable; EGTA, ethylene glycol tetraacetic acid; FL, full-length; NT, N-terminal deletion; CaM, calcium/calmodulin binding domain; RyR, ryanodine receptor, gf, gain-of-function mutation; CER, ceramide; CIB, calcium and integrin binding protein. ${ }^{*} p<0.05,{ }^{* *} p<0.005$, ${ }^{* * *} p<0.0005$.

$\sin ($ Fig. $1 b)$. In body wall muscles, SPHK-1-GFP was primarily observed on the outer surface of mitochondria as demonstrated by its colocalization with an RFP-tagged outer membrane mitochondrial marker (Fig. 1c). Thus, SPHK-1 appears to localize to different cellular membranes depending on cell type.

\section{Synaptic recruitment of SPHK-1 is regulated by presynaptic calcium}

Previous studies have found that neuronal depolarization or hyperpolarization results in corresponding decreases or increases in SphK synaptic abundance (Kajimoto et al., 2007; Chan et al., 2012). To explore whether calcium influx into motor neurons mediates the effects of neuronal activity on SPHK-1 synaptic recruitment, we examined SPHK-1-GFP in animals with defects in presynaptic calcium entry. To detect changes in synaptic SPHK-1-GFP fluorescence, we examined fluorescence intensity and distribution of SPHK-1-GFP puncta in DA/DB class motor axons. Among the parameters examined were punctal fluorescence, which measures synaptic abundance; punctal width, which measures distribution within a synapse; and punctal density, which measures the number of puncta per unit length of an axon.

Calcium enters synaptic terminals from two main sources: the extracellular space and internal stores. We first tested the effects of mutations that alter calcium entry through voltage-gated calcium channels (VGCCs). unc-2 encodes the C. elegans $\alpha 1$ subunit of the non-L type voltage-gated calcium channel (VGCC $\alpha 1$ ). UNC-2/VGCC $\alpha 1$ localizes to presynaptic active zones, and unc-2/VGCC $\alpha 1$ mutations reduce calcium influx in certain sensory neurons (Richmond and Jorgensen, 1999; Mathews et al., 2003; Saheki and Bargmann, 2009). We found that loss-of-function mutations in $u n c-2 / \mathrm{VGCC} \alpha 1$ reduced
SPHK-1 average punctal fluorescence by $\sim 20 \%$ (Fig. $2 a$; Table $1)$. The decrease in punctal fluorescence was due to a reduction in the fraction of puncta with high fluorescence intensities and a corresponding increase in the fraction of puncta with low intensities, as revealed by cumulative probability plots (Fig. $2 b$ ). In contrast, unc-2/VGCC $\alpha 1$ mutations did not significantly alter either SPHK-1-GFP punctal width or punctal density, indicating that $u n c-2 / V G C C \alpha 1$ specifically affects SPHK-1 abundance, possibly by regulating its translocation to synaptic terminals. unc-2/ VGCC $\alpha 1$ does not impact overall synaptic structure since the punctal fluorescence of active and periactive zone proteins (e.g., UNC-10/RIM1, SYD-2/liprin- $\alpha$, SNN-1/synapsin, and ITSN-1/intersectin) are not significantly altered in unc-2/VGCC $\alpha 1$ mutants (Ch'ng et al., 2008). unc2/VGCC $\alpha 1$ mutations severely reduce acetylcholine release from motor neurons and dramatically alter synaptic vesicle cycling (Richmond et al., 2001; Ch'ng et al., 2008). Thus, it is possible that the decreases observed in SPHK-1 synaptic abundance are a secondary effect of alterations in synaptic vesicle cycling or release in these mutants. This is likely not to be the case because synaptic SPHK-1-GFP abundance is not detectably altered in $u n c-13 /$ Munc13 or unc18/Munc18 mutants (Chan et al., 2012), in which synaptic vesicle release and cycling are blocked but calcium entry remains intact (Richmond et al., 2001; Arellano-Carbajal et al., 2011). Thus calcium entry through UNC-2/VGCC $\alpha 1$ positively regulates SPHK-1 synaptic abundance independently of its effects on synaptic vesicle release.

To test whether calcium plays a permissive or instructive role in SPHK-1 recruitment, we examined egl-19, which encodes the $\alpha 1$-subunit of the $C$. elegans L-type voltage-gated calcium channel (L-VGGC $\alpha 1$ ). egl-19/L-VGGC $\alpha 1$ is expressed in motor neurons of the ventral nerve cord (Hunt-Newbury et al., 
2007), and egl-19/L-VGGC $\alpha 1$ loss-offunction mutants have reduced calcium influx, whereas egl-19/L-VGGC $\alpha 1$ gainof- function mutants have increased calcium entry (Kerr et al., 2000; Frøkjaer-Jensen et al., 2006). We observed a $20 \%$ decrease and 34\% increase in SPHK-1 abundance in egl-19/L-VGGC $\alpha 1$ loss-of-function and gain-of-function mutants, respectively, compared with wild-type controls (Fig. 2a,b; Table 1). The increase in SPHK-1-GFP fluorescence in egl-19/LVGGC $\alpha 1$ gain-of-function mutants was blocked by acute treatment with the calcium chelator EGTA (Fig. 2c). Thus, egl-19/L-VGGC $\alpha 1$ activation elicits a calcium-dependent increase in synaptic SPHK-1-GFP due to acute changes in calcium influx. Together, these results indicate that calcium influx is both necessary and sufficient for SPHK-1 recruitment, and suggest that calcium is instructive in this process.

We next examined the contribution of calcium entry through calcium stores to SPHK-1 recruitment. unc-68 encodes a ryanodine receptor (RyR), which mediates calcium efflux from intracellular calcium stores. unc-68/RyR mutants have reductions in neurotransmitter release from motor neurons (Liu et al., 2005). We found that mutations that reduce the activity of unc-68/ RyR significantly reduced SPHK-1-GFP peak fluorescence compared with wild-type controls (Fig. 2a, $b$; Table 1), without affecting either punctual width or density (Table 1). Thus, it appears that calcium from both intracellular and extracellular sources is needed for SPHK-1 recruitment.

\section{Calcium entry is required for muscarinic-dependent SPHK-1 recruitment}

Activating a presynaptic muscarinic pathway either by acute treatment of $C$. elegans with the muscarinic agonist arecoline or by constitutively activating the heterotrimeric G protein G $\alpha q(e g l-30 / G \alpha q(g f))$ significantly increases SPHK-1-GFP punctal fluorescence (Fig. 2d,e; Table 1) (Chan et al., 2012), suggesting that muscarinic signaling regulates SPHK-1 translocation to release sites. We examined whether muscarinic-regulated translocation of SPHK-1 is dependent on calcium influx. The increase in SPHK1-GFP punctal fluorescence caused by either arecoline or by a constitutively active egl-30/G $\alpha q$ mutant was completely blocked by $u n c-2 /$ VGCC $\alpha 1$ mutations (Fig. $2 d$,e; Table 1 ). These results suggest that presynaptic calcium is required for muscarinicmediated recruitment of SPHK-1 to synapses.

Arecoline treatment causes animals to become hypersensitive to the paralytic effects of the AChE inhibitor aldicarb (Lackner et al., 1999). Aldicarb prevents removal of acetylcholine from the synaptic
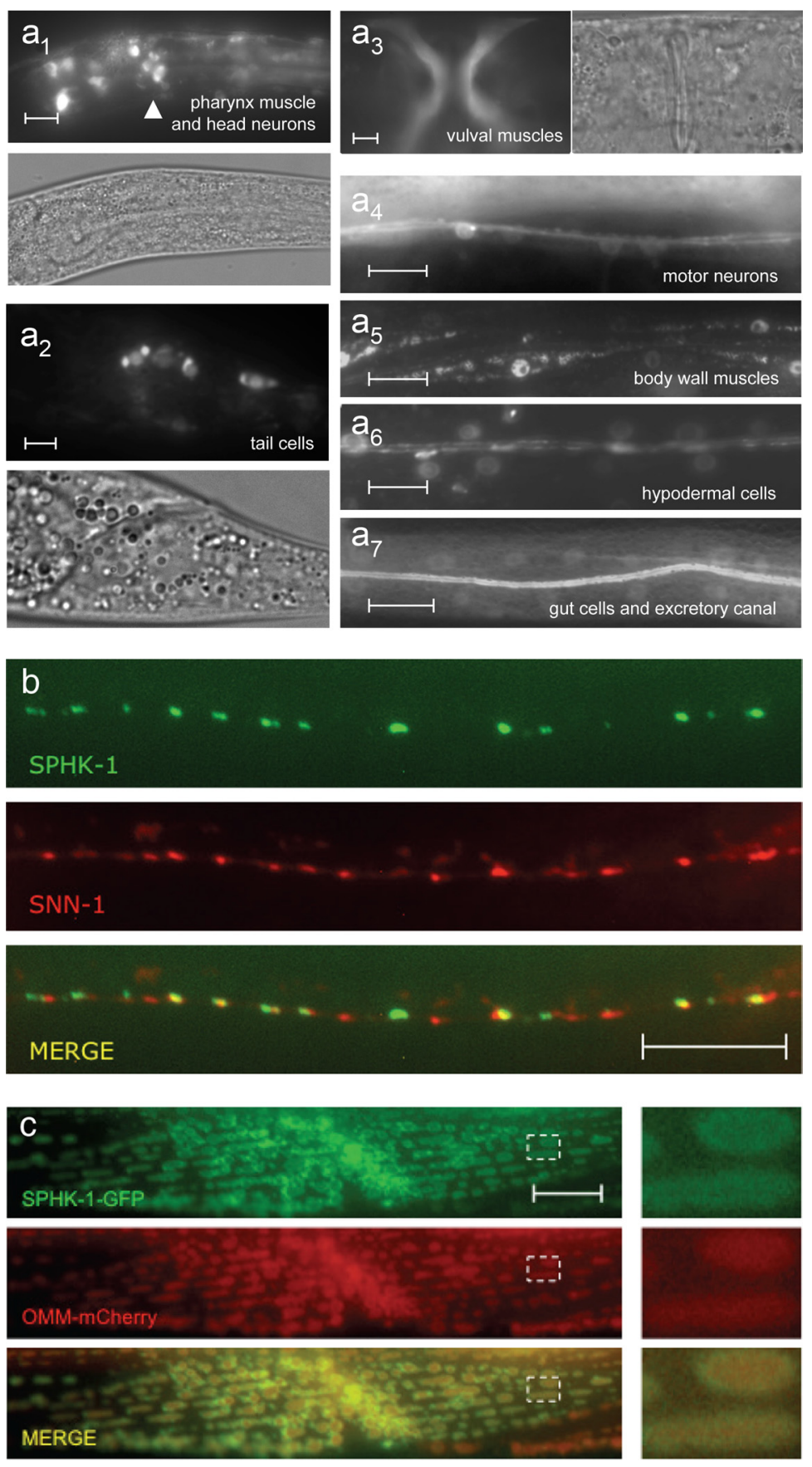

Figure 1. Expression and localization of SphK in C. elegans. $\boldsymbol{a}$, Expression of an sphk-1 promoter fragment driving either GFP $\left(a_{3}\right.$ $a_{4}$, and $\left.a_{7}\right)$ or nuclear localized GFP $\left(a_{1}, a_{2}, a_{5}\right.$, and $\left.a_{6}\right)$ in adults. Analogous DIC images are also shown for $a_{1}-a_{3}$. Arrowhead indicates neurons in the nerve ring. $\boldsymbol{b}$, Representative images showing the localization of SPHK-1-GFP with SNN-1/synapsin (SNN-1-mCherry). c, Representative images showing the localization of SPHK-1-GFP and an outer mitochondrial membrane marker (0MM-mCherry) in body wall muscles (expressed under the myo-3 promoter). The dotted box was magnified $4 \times$ and shown to the right of the images. Scale bars: $10 \mu \mathrm{m}$.

cleft, causing muscle hypercontraction and paralysis. Hypersensitivity to aldicarb is observed under conditions of increased neuromuscular function, including enhanced neurotransmitter release (Mahoney et al., 2006; Vashlishan et al., 2008). sphk-1 is required for the effects of arecoline treatment on aldicarb hypersensitivity (Chan et al., 2012), suggesting that sphk-1 mediates the effects of arecoline on synaptic transmission. If the recruitment of SPHK-1 by presynaptic calcium was required for the effects of muscarinic signaling on synaptic transmission, then we would expect calcium mobilization 

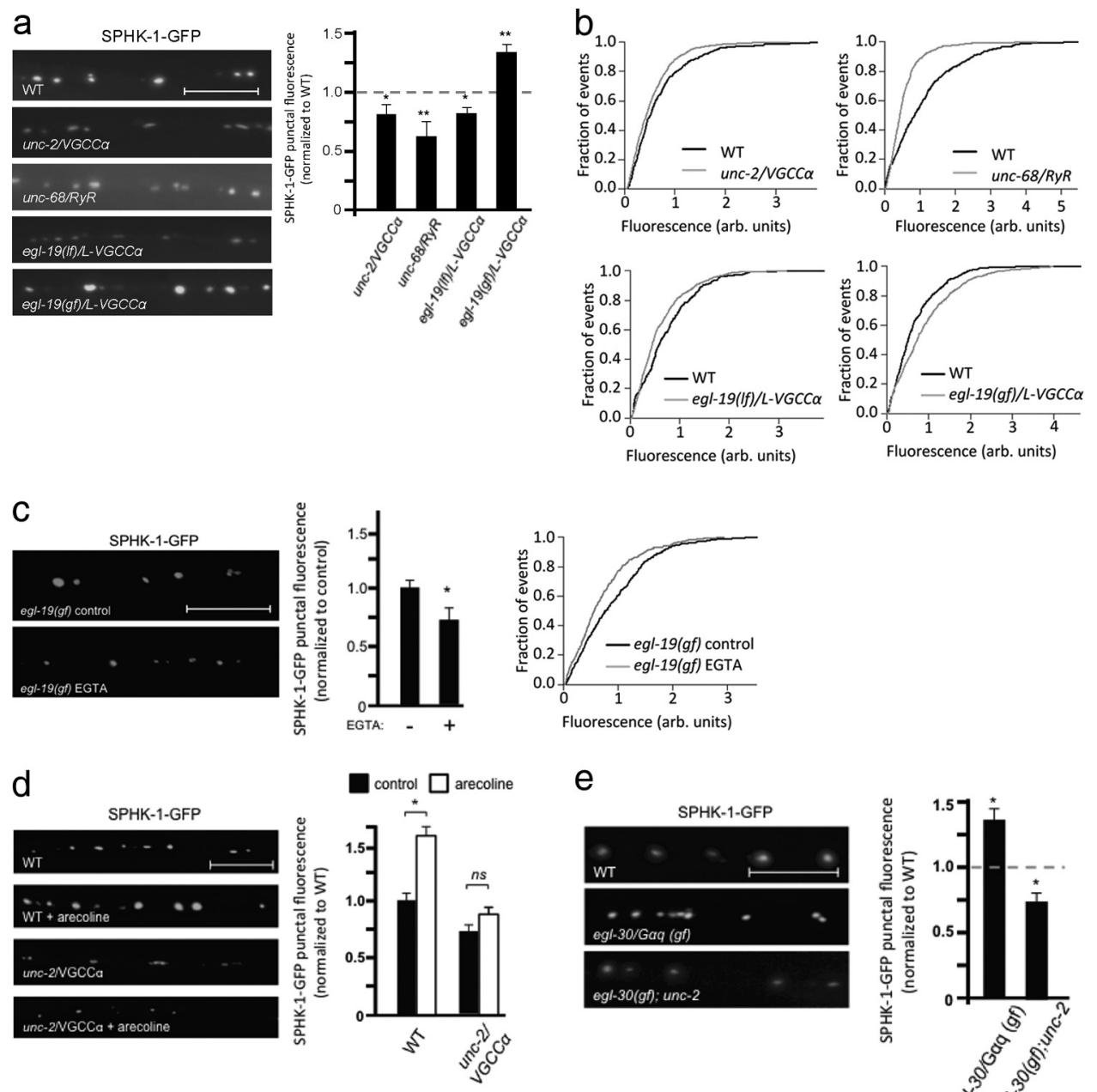

e
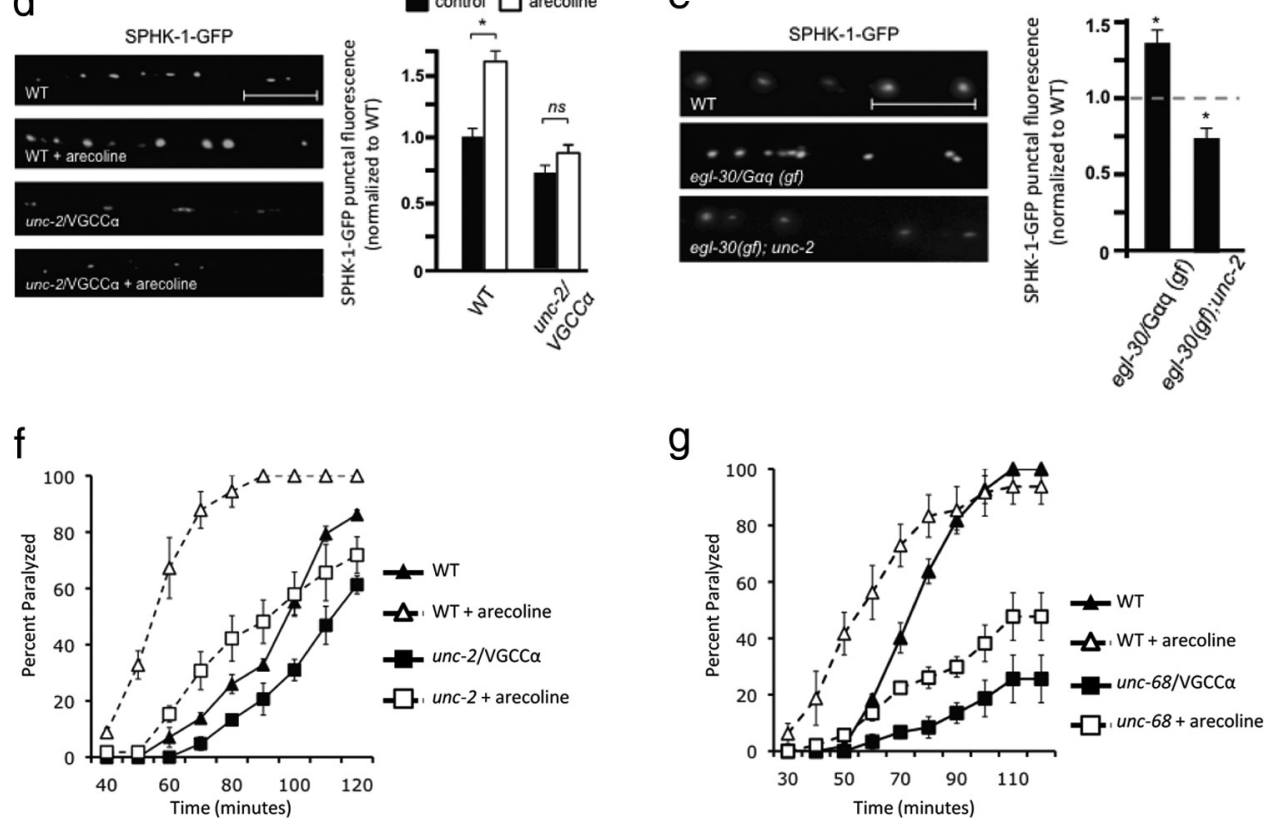

Figure 2. Calcium mediates the basal and muscarinic-mediated recruitment of SPHK-1 at synapses. $\boldsymbol{a}$, Representative images (left) and quantification (right) of SPHK-1-GFP punctal fluorescence in the indicated mutants. $\boldsymbol{b}$, Cumulative probability plots showing the distribution of SPHK-1-GFP intensities per punctum. c, Representative images (left), quantification (middle), and cumulative probability plot (right) of SPHK-1-GFP punctal fluorescence in egl-19/L-VGCC $\alpha 1$ gain-of-function mutants, treated with water, pH 7.2, or EGTA (50 mM, pH 7.2). d, e, Representative images (left) and quantification (right) of SPHK-1-GFP punctal fluorescence in the indicated mutants. The indicated strains were analyzed following a $2 \mathrm{~h}$ treatment with M9 (salt solution, black bars) or arecoline (15 mm in M9; white bars) and the data are normalized to wild-type (WT; control) fluorescence. $\boldsymbol{f}, \boldsymbol{g}$, Rates of worm paralysis of the unc-2NGCC $\alpha 1(\boldsymbol{f})$ or unc-68/RyR $(\boldsymbol{g})$ mutants upon exposure of the AChE inhibitor aldicarb (1.0 mM) following a $2 \mathrm{~h}$ pretreatment with control M9 (solid lines) or arecoline (15 mm in M9; dotted lines). For all, ${ }^{*} p<0.05$ and ${ }^{* *} p<0.005$, Student's t tests. Scale bars: $10 \mu \mathrm{m}$.

mutants to block the aldicarb hypersensitivity caused by arecoline treatment. Consistent with this idea, we found that $u n c-2 / \mathrm{VGCC} \alpha 1$ or unc-68/RyR mutants had a severely blunted response to arecoline treatment compared with wild-type animals, indicating that the effects of muscarinic stimulation on aldicarb responsiveness are partially dependent on calcium entry. (Fig. $2 f, g$ ). Together these results suggest that presynaptic calcium promotes both basal and muscarinic-potentiated synaptic transmission by recruiting SPHK-1 to presynaptic terminals.

\section{CALM-1/CIB is presynaptic and mediates \\ SPHK-1 recruitment}

We next examined the molecular mechanism by which SPHK-1 might be recruited to synapses by calcium. SphKs do not contain sequence motifs that are known to bind calcium directly. However, SphKs contain a conserved calcium/CaM binding motif that has been shown to interact with calcium/CaM as well as CIB (Young et al., 2003; Jarman et al., 2010). CIB is a family of calcium responsive proteins with diverse biological functions, including 
cell survival, growth, migration, and synaptic plasticity (Kauselmann et al., 1999; Leisner et al., 2005; Yoon et al., 2009; Frost and Olson, 2010). CIB1 recently has been implicated in the calcium-dependent recruitment of human SphK1 to the plasma membrane in HEK cells (Jarman et al., 2010). C. elegans encodes an ortholog to the CIB family, calm-1, which shares 57\% similarity to mammalian CIB1 and $48 \%$ similarity to mammalian CIB2. calm-1 mRNA is expressed in motor neurons (Fox et al., 2005). We found that a calm-1 transcriptional reporter, in which 1852 bp of the calm-1 promoter drove the expression of GFP, was expressed in body wall muscle and in cholinergic motor neurons of the ventral cord (Fig. 3a). We examined the subcellular localization of a CALM-1/CIB fusion protein (CALM1 -mCherry) in motor neuron axons and found that it adopted a punctate pattern of fluorescence, that largely colocalized with SPHK-1-GFP puncta at presynaptic terminals (Fig. $3 b$ ).

Next, we examined the requirement of calm-1/CIB in regulating SPHK-1 synaptic recruitment. calm-1(tm1353) mutants, which have a 618 nucleotide deletion that removes part of the calm-1 promoter and the first exon, showed a significant decrease in SPHK-1-GFP synaptic abundance (Fig. $3 c$; Table 1), without altering SPHK-1 abundance in neuronal cell bodies (336 \pm 45 arbitrary fluorescence units in WT vs $342 \pm 38$ units in calm-1/CIB mutants, $p>0.05)$. We also found that arecoline-mediated recruitment of SPHK-1GFP to synapses was blocked in calm-1/ CIB mutants (Fig. 3d; Table 1). Together these results suggest that presynaptic CALM-1 promotes the recruitment of SPHK-1 to release sites.

The calcium/CaM-binding domain of SPHK-1 is required for muscarinicmediated SPHK-1 recruitment

To investigate how calcium and muscarinic signaling might converge to regulate SPHK-1 abundance, we next sought to identify the SPHK-1 sequence determinants that are responsible for the synaptic translocation of SPHK-1 in response to calcium or muscarinic signaling. SPHK-1 contains five conserved domains, termed $\mathrm{C} 1-\mathrm{C} 5$, which share $>50 \%$ amino acid similarity with human SphK1 and SphK2 (Fig. 4a). We examined the synaptic abundance of GFP-tagged SPHK-1 variants in which various conserved domains had been deleted (Fig. 4a). An SPHK1-GFP variant that contains only the kinase domain (SPHK$1(\mathrm{NT} 1)$ ) adopted a diffuse pattern of fluorescence in axons and in cell bodies (Fig. 4a,b), implying a redistribution of the protein to the cytosol (Hengst et al., 2010; Siow et al., 2011). Quantification of the localization pattern of the kinase domain of SPHK-1 in axons revealed a reduction in punctal fluorescence and a corre-

a

d
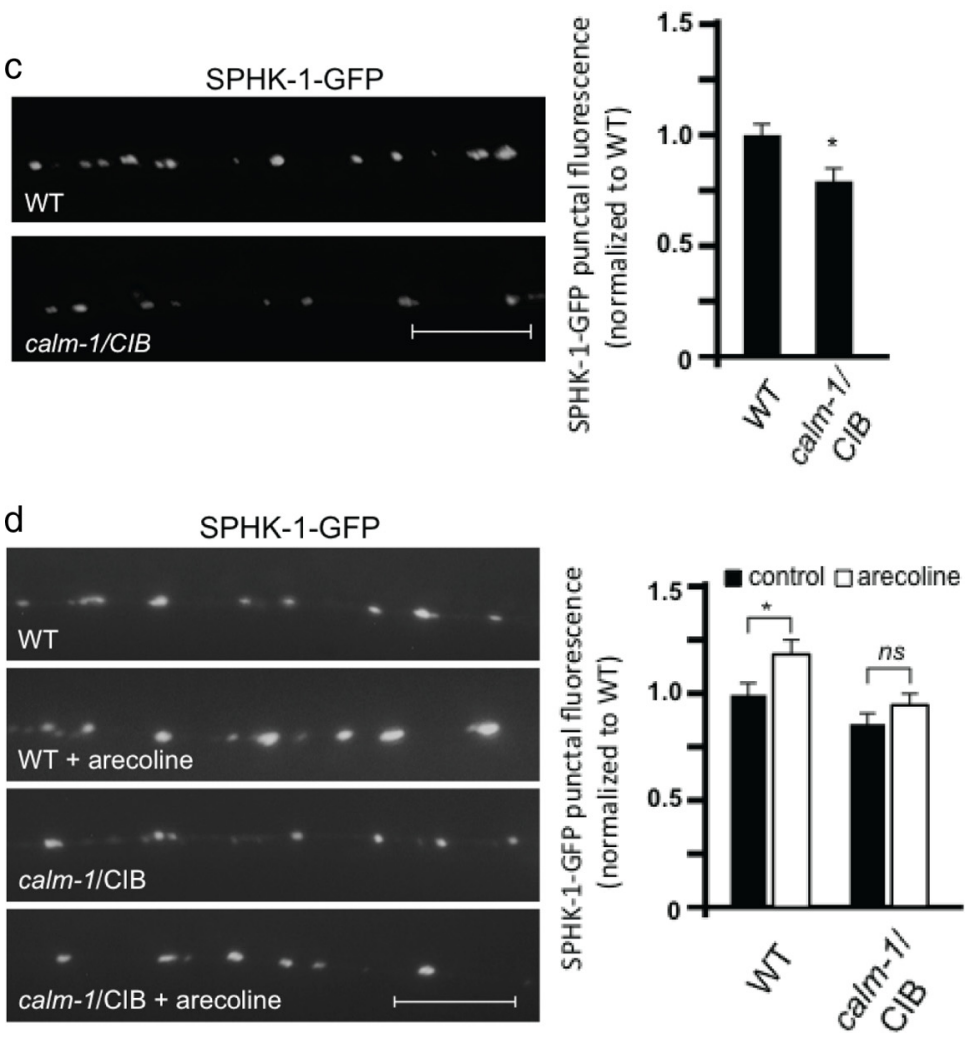

Figure 3. Muscarinic-mediated recruitment of SPHK-1 at synapses depends on CALM-1/CIB. $\boldsymbol{a}$, Fluorescence images showing GFP expressed under the calm-1 transcriptional reporter (P calm-1-gfp) in neurons of the ventral cord, which colocalize with mCherry expressed 列 CIB-1 (CALM-1-mCherry) in DA/DB motor neurons (both using the unc-129 promoter). Arrows indicate regions of colocalization and arrowheads indicate regions where CALM-1-mCherry was observed without SPHK-1-GFP. c, Representative images (left) and quantification (right) of SPHK-1-GFP punctal fluorescence in WT or calm-1/CIB mutants following pretreatment with control M9 (salt solution, black bars) or arecoline (15 mm in M9; white bars). Scale bars: (for $\boldsymbol{a}) 5 \mu \mathrm{m}$; (for $\boldsymbol{b}-\boldsymbol{d}$ ) $10 \mu \mathrm{m}$.

sponding increase in interpunctal fluorescence compared with wild-type SPHK-1-GFP (Fig. 4c).

Addition of the $\mathrm{N}$ terminus of SPHK-1 to the kinase domain (SPHK-1(NT2)) restored both synaptic localization and presumed Golgi localization (Fig. 4a,b). However, SPHK-1(NT2)GFP synaptic puncta were significantly dimmer and wider than wild-type controls, and fluorescence was also found on the plasma membrane in cell bodies in three of three lines we examined (Fig. 4b,c; Table 1). Adding the conserved domain C4 to this variant (SPHK-1(NT3)) restored wild-type levels of SPHK-1 at synapses and restored Golgi localization in cell bodies (Fig. 4a,b). However, synaptic puncta remained significantly wider than wild-type controls (Fig. 4b; Table 1). A 110 aa fragment (SPHK- 


\section{a}

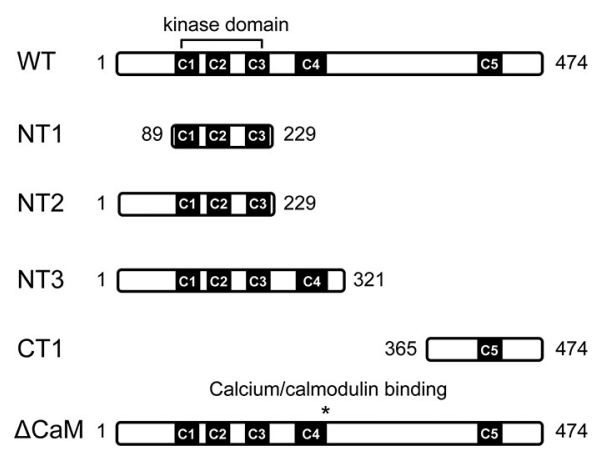

b

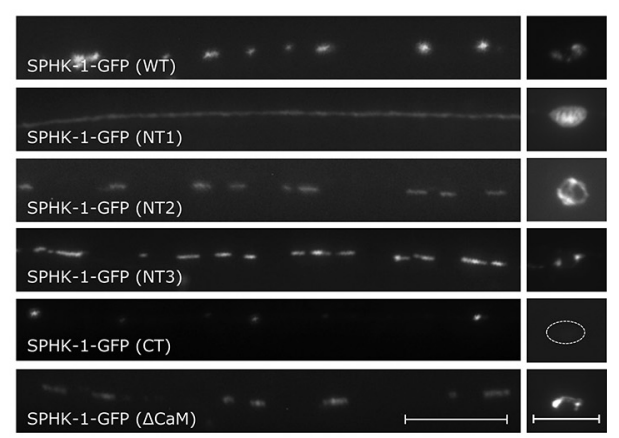

d

c

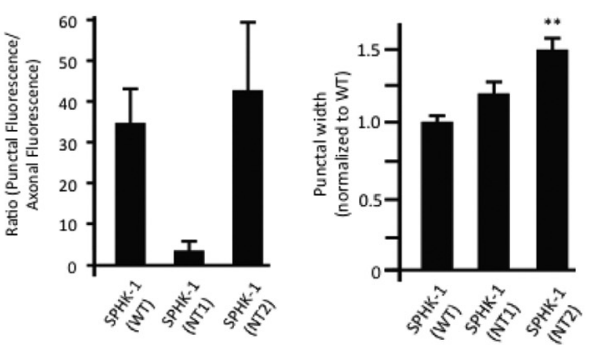

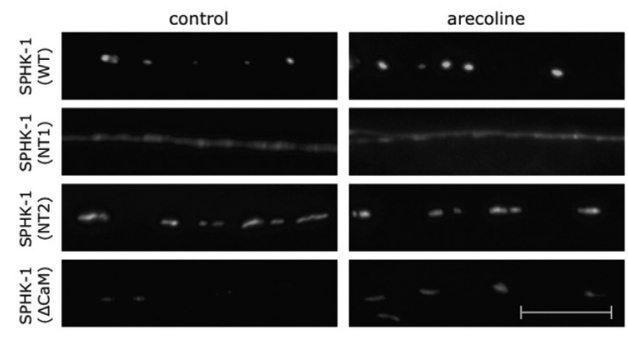

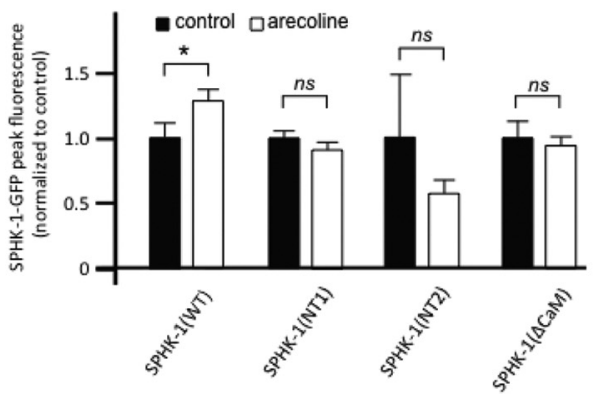

Figure 4. Structural determinants that localize SPHK-1 at synapses and cell bodies. $\boldsymbol{a}$, Diagram of wild-type(WT) SPHK-1-GFP and its variants used. $\boldsymbol{b}$, Representative images of the localization of SPHK-1-GFP and indicated variants in DA/DB motor neuron axons (left) and cell body (right).c, Quantification of the ratio of punctal fluorescence to interpunctal fluorescence (left) and punctal width (right) of SPHK-1(WT)GFP, SPHK-1(NT1)-GFP, and SPHK-1(NT3)-GFP in DA/DB motorneurons.d, Representative images (top) and quantification (bottom) ofSPHK-1-GFP (WT and the indicated variants) in DA/DB motor neurons with pretreatment of control M9 (black bars) or arecoline (15 mm in M9; white bars). For all, ${ }^{*} p<0.05$ and ${ }^{* *} p<0.005$, Student's t tests. Scale bars: $10 \mu \mathrm{m}$.

$1(\mathrm{CT} 1))$ formed puncta in axons that were similar in size to SPHK-1(WT)-GFP puncta. However, these puncta were much less frequent compared with SPHK-1(WT)-GFP, and SPHK1(CT1) was not detectable in cell bodies (Fig. 4a,b). Finally, a full-length SPHK-1 variant containing a mutation in the $\mathrm{C} 4$ domain that corresponds to the calcium/CaM binding domain (SPHK-1 $(\Delta \mathrm{CaM})$-GFP) adopted a pattern that was similar to SPHK-1(NT2): wider and dimmer synaptic puncta and mislocalization in cell bodies (Fig. $4 a, b$; Chan et al., 2012). Together, these results suggest that the region $\mathrm{N}$-terminal of the kinase domain can direct SPHK-1 to synapses (and to Golgi), but does not restrict SPHK-1 within synapses. Similarly, the C4 domain does not appear to restrict SPHK-1 to small regions within synapses; however, it is required for normal SPHK-1 levels at synapses. On the other hand, the similarity of the localization patterns SPHK1 (NT2) and the SPHK-1 $(\Delta \mathrm{CaM})$ variants, both of which lack the CaM domain in C4, suggests that the CaM domain is essential for recruiting and restricting SPHK-1 within synapses. However, the C4 domain is likely to not be sufficient for synaptic clustering of SPHK-1 since SPHK-1(NT3), which has the C4 domain, fails to cluster. Thus, SPHK-1 may require both the C4 and the C5 domains to restrict SPHK-1 within synapses.
To identify the sequence determinants required for muscarinicmediated SPHK-1 synaptic recruitment, we next tested the effects of acute arecoline treatment $(2 \mathrm{~h})$ on the abundance of the SPHK-1 variants at synapses. Although arecoline treatment significantly increased the punctal fluorescence of SPHK-1-GFP transgenes, arecoline treatment had no effect on the distribution or abundance of the SPHK-1(NT1)-GFP, SPHK-1(NT3)-GFP, or SPHK-1(CaM)-GFP variants (Fig. $4 d$; Table 1). These results suggest that sequence determinants that lie C-terminal to the $\mathrm{C} 4$ domain as well as the CaMbinding site are important for arecoline responsiveness. Our observation that either calm-1/CIB mutants or deletion of the CaMbinding site block muscarinic-mediated SPHK-1 translocation suggests that CALM-1/CIB acting on the CaM-binding site of SPHK-1 may be critical for the ability of muscarinic signaling to recruit SPHK-1 to synapses.

HYL-1/ceramide synthase and TAG-38/S1P lyase are concentrated at presynaptic terminals

Multiple enzymes catalyze the generation and degradation of sphingolipids, and they are thought to function coordinately to fine-tune levels of each sphingolipid metabolite. If the regulation of synaptic levels of SPH and S1P by SPHK-1 is important for 
synaptic function, then we expect that additional enzymes involved in SPH or S1P metabolism might also function at synapses. To test this idea, we examined two enzymes that are predicted to directly affect levels of SPH and S1P: ceramide (CER) synthase, which converts SPH to CER, and S1P lyase, which breaks down S1P into phosphoethanolamine and hexadecanal (Fig. 5a). C. elegans encodes three predicted CER synthases. One of these, $h y l-1$, is expressed in motor neurons (Fox et al., 2005), and mutants lacking hyl-1 display hyperactive locomotion (Chan et al., 2012). We generated a GFP-tagged HYL-1 fusion protein (HYL-1-GFP) and found that it adopted a punctate pattern of fluorescence in motor neuron axons (Fig. 5b). HYL-1-GFP largely colocalized with the SV marker SNB-1-mCherry (Fig. $5 d$ ), but was mostly apposed to SPHK-1mCherry and SNN-1-mCherry puncta (Fig. 5b,d). HYL-1-GFP puncta were eliminated in mutants lacking unc-104/ kinesin, which encodes a motor protein that transports SVs and other organelles to presynaptic terminals (Fig. $5 c$ ). These results suggest that HYL-1 associates with synaptic vesicle pools (or other presynaptic organelles) at presynaptic terminals.

C. elegans encodes two predicted S1P lyases, spl-1 and tag-38. Whereas spl-1 expression has not been detected in the nervous system (www.wormbase.org), tag-38 cDNA is expressed in motor neurons (Fox et al., 2005). Therefore, we examined a GFP-tagged TAG-38/S1P lyase fusion protein (TAG-38-GFP) and found that it adopted a highly punctate pattern of fluorescence in axons that largely overlapped with SPHK-1-mCherry or SNN-1-mCherry puncta (Fig. 5b,d). TAG-38-GFP localized less well with the SV markers SNB-1 and remained highly punctate in $u n c-104 / \mathrm{ki}-$ nesin mutants (Fig. $5 c$ ). Together these results suggest that like SPHK-1, TAG-38/ S1P lyase associates with presynaptic membranes at release sites, but not synaptic vesicles. Given the striking similarity between the localization of TAG-38/S1P lyase and SPHK-1, we wondered whether TAG-38/S1P lyase synaptic abundance might also be regulated by muscarinic signaling. However, we found that arecoline treatment did not significantly change TAG-38-GFP axonal punctal fluorescence, width, or density (Fig. 5e; Table 1). Therefore, muscarinic signaling appears to specifically regulate SPHK-1 abundance, but not TAG-38/S1P lyase, at release sites.

\section{hyl-1/ceramide synthase antagonizes $s p h k-1$}

We previously showed that $h y l-1 / C E R$ synthase mutants have hyperactive locomotion rates compared with wild-type controls (Chan et al., 2012). To examine the role of hyl-1/CER synthase in synaptic

b

C

d

\section{a Sphingolipid Metabolism}
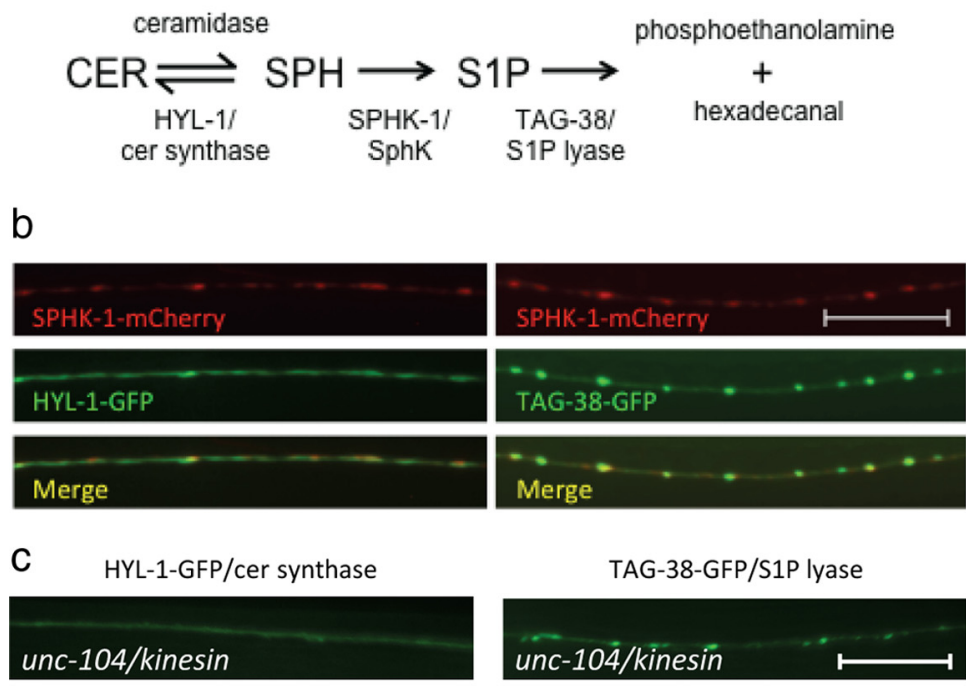

TAG-38-GFP/S1P lyase
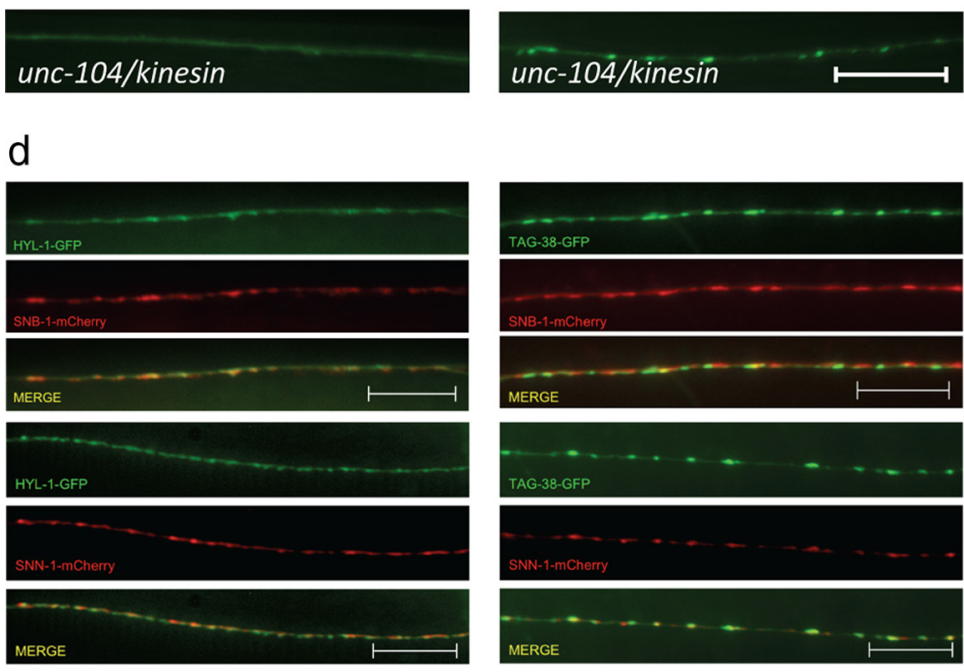

e

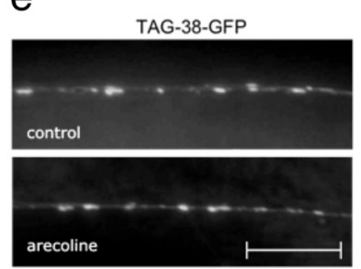

Figure 5. Localization of HYL-1/CER synthase and TAG-38/S1P lyase at synapses. $\boldsymbol{a}$, Diagram of the sphingolipid metabolism pathway and its enzymes that have orthologs in C. elegans. CER, ceramide; SPH, sphingosine; S1P, sphingosine-1-phosphate. $\boldsymbol{b}$, Representative images showing the localization of SPHK-1-mCherry with HYL-1-GFP (left) or TAG-38-GFP (right) in DA/DB motor neurons. c, Representative image of HYL-1-GFP (left) or TAG-38-GFP (right) at synapses of DA/DB motor neurons in mutants lacking the synaptic vesicle trafficking protein unc-104/Kinesin KIF1A. $\boldsymbol{d}$, Representative images showing the localization of HYL-1-GFP (left) or TAG-38-GFP (right) with SNB-1/synaptobrevin (mCherry-SNB-1; top) and SNN-1/synapsin (SNN-1-mCherry; bottom) in DA/DB motor neurons. $\boldsymbol{e}$, Representative images (top) and quantification (bottom) of TAG-38-GFP in DA/DB motor neurons following pretreatment of control M9 (salt solution, black bars) or arecoline (15 mm in M9; white bars). Scale bars: $10 \mu \mathrm{m}$.

transmission more directly, we examined aldicarb responses and SV cycling in $h y l-1 /$ CER synthase mutants. We found that $h y l-1 / \mathrm{CER}$ synthase mutants where hypersensitive to aldicarb (Fig. 6a), consistent with increased ACh release at neuromuscular junctions. hyl1/CER synthase mutants also displayed increases in punctual fluorescence of the SV marker SNB-1-GFP (Fig. 6b; Table 1). Increases in punctal fluorescence are indicative of larger SV pools at synaptic terminals, and may arise from alterations in SV trafficking, biogenesis, or release. The increase in locomotion rates, the aldicarb hypersensitivity, and the increase in SV abundance of 

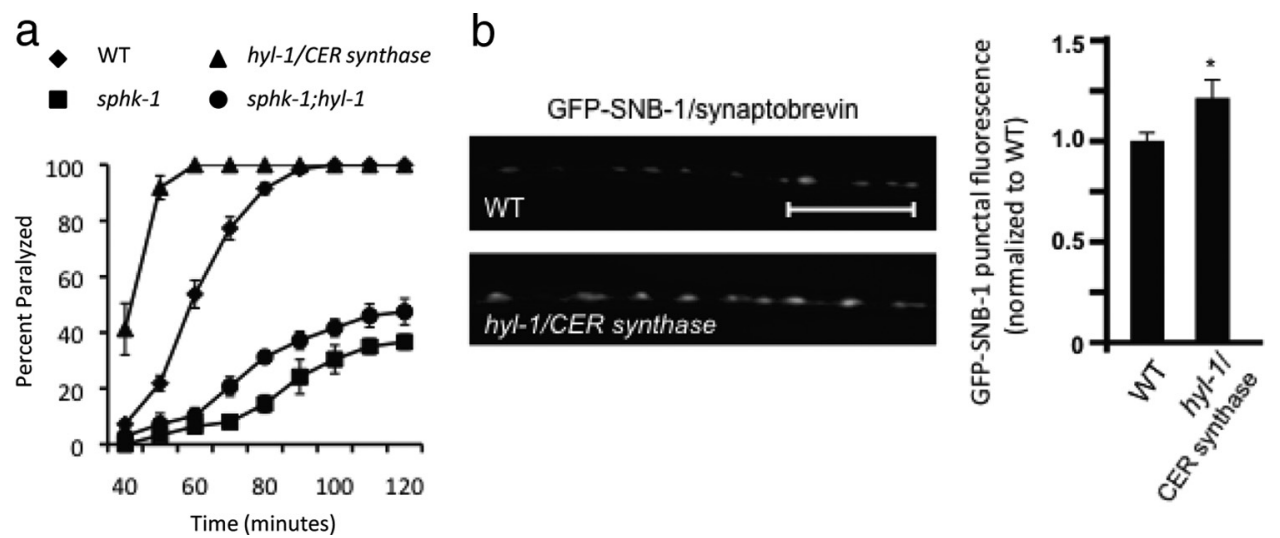

Figure 6. HYL-1/ceramide synthase antagonizes SPHK-1.a, Rates of worm paralysis of wild-type (WT) controls and hyl-1/CER synthase mutants upon exposure to the AChE inhibitor aldicarb (1.0 $\mathrm{mm}$ ). $\boldsymbol{b}$, Representative images (left) and quantification (right) of GFP-tagged synaptobrevin (GFP-SNB-1) in axons of DA/DB motor neurons analyzed in the WT and hyl-1/CER synthase mutants. ${ }^{*} p<0.05$, Student's $t$ tests. Scale bar, $10 \mu \mathrm{m}$.

hyl-1/CER synthase mutants are similar to those observed in constitutively active e $g l-30 / G \alpha q(g f)$ mutants (Ch'ng et al., 2008). In-

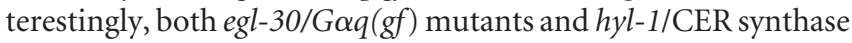
mutants are predicted to have elevated S1P levels: egl-30/ $G \alpha q(g f)$ mutants have more SPHK-1 at synapses (Chan et al., 2012), and hyl-1/CER synthase mutants have fivefold higher levels of the S1P precursor SPH (Menuz et al., 2009). Thus, elevated S1P levels may account for the similar phenotypes observed in these mutants.

If elevated synaptic S1P promotes synaptic transmission, then we would predict that the aldicarb hypersensitivity of $h y l-1 \mathrm{mu}-$ tants should be dependent on S1P production by SPHK-1. Indeed, we found that sphk-1;hyl-1 double mutants were nearly as aldicarb resistant as sphk-1 single mutants (Fig. 6a). Together, these data suggest that high SPH levels alone cannot account for the increased locomotion rates, aldicarb hypersensitivity, and accumulation of SVs in $h y l-1 / C E R$ synthase mutants. Instead, these observations indicate that the conversion of SPH to S1P by SPHK-1 is important for neurotransmission.

\section{sphk-1 is required for muscarinic-mediated recruitment of UNC-13S to synapses}

How might synaptic SPHK-1 couple to the SV release machinery to promote acetylcholine release? Munc13 is a presynaptic protein involved in priming of secretory vesicles through its interaction with the SNARE protein syntaxin (Brose et al., 2000; Madison et al., 2005). The association of Munc13 with release sites is regulated by diacylglycerol (DAG) and is proposed to control vesicle priming and subsequent neurotransmitter release (Richmond et al., 1999; Madison et al., 2005). Like SPHK-1, C. elegans Munc13, UNC-13, is recruited to presynaptic terminals by EGL-30/G $\alpha$ q-dependent muscarinic signaling (Lackner et al., 1999). We previously found that SPHK-1-GFP punctal fluorescence did not change in unc-13/Munc13 mutants (Chan et al., 2012), suggesting that UNC-13/Munc13 is not required for SPHK-1 synaptic recruitment.

We next examined whether the synaptic abundance of GFP-tagged UNC-13 (UNC-13S-GFP) was dependent on sphk-1 activity. We found no change in synaptic abundance of UNC-13S-GFP in sphk-1 mutants compared with wild-type controls under normal conditions (Fig. $7 a$ and Table 1). However, sphk-1 activity was essential for muscarinic signalingdependent UNC-13S-GFP recruitment. Activating muscarinic signaling (using gain-of-function egl-30/G $\alpha \mathrm{q}$ mutants) signifi- cantly increased UNC-13S-GFP punctal fluorescence (Fig. 7a; Table 1). egl-30/G $\alpha \mathrm{q}$ activation appears to specifically recruit UNC-13S-GFP to synapses because egl-30/G $\alpha$ q mutations did not significantly alter the punctal fluorescence of another active zone protein, UNC-10/RIM1 (Ch'ng et al., 2008). The increase in UNC-13S-GFP punctal fluorescence was completely blocked by sphk-1 mutations: punctal UNC-13S-GFP fluorescence was reduced to wild-type levels in egl-30(gf);sphk-1 double mutants (Fig. 7a; Table 1). Thus, muscarinic signaling may selectively recruit UNC-13S to synaptic membranes via an sphk-1dependent mechanism (Fig. 7b).

\section{Discussion}

Changes in presynaptic structure and function represent important mechanisms by which modulatory pathways regulate synaptic strength. In this study, we found that muscarinic signaling regulates the abundance of SPHK-1 at synaptic terminals via a calcium-dependent mechanism involving CIB. Synaptic SPHK-1 in turn promotes the recruitment of at least one priming factor, UNC-13/Munc13, in response to muscarinic signaling. Thus, local sphingolipid metabolism appears to be an important way by which synaptic vesicle priming is regulated.

\section{Calcium mobilization regulates the abundance of SPHK-1 at synapses}

We found that calcium mobilization regulates SPHK-1 synaptic abundance and is required for SPHK-1 translocation in response to muscarinic signaling. First, mutants with reduced extracellular calcium influx through non-L or L-VGGC (unc-2/VGCC $\alpha 1$ or egl-19/L-VGCC $\alpha 1$ ) or reduced intracellular calcium influx through ryanodine receptors (unc-68/RyR) decreased steadystate synaptic SPHK-1 abundance. Second, increasing calcium influx (in egl-19/L-VGCC $\alpha 1$ gain-of-function mutants) increased synaptic SPHK-1 abundance in a calcium-dependent manner. Interestingly, the changes in SPHK-1 synaptic abundance we observed in egl-19 loss- and gain-of function mutants (18\% decrease and $34 \%$ increase) correlate with the changes in calcium influx observed in the respective mutants (19\% decrease and 19\% increase) (Kerr et al., 2000). Third, calcium influx through unc-2/VGCC $\alpha 1$ was necessary for muscarinic-mediated recruitment of SPHK-1 to synapses and for muscarinic-induced aldicarb hypersensitivity. Fourth, SPHK-1 variants lacking only the calcium/CaM-binding domain of SPHK-1 were mislocalized at synapses and were not responsive to synaptic recruitment by 
a

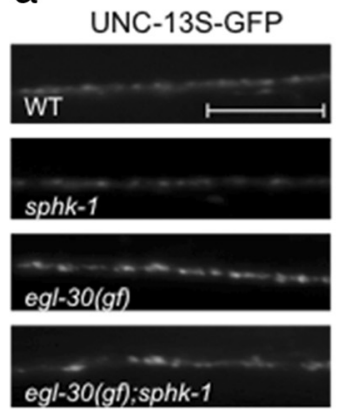

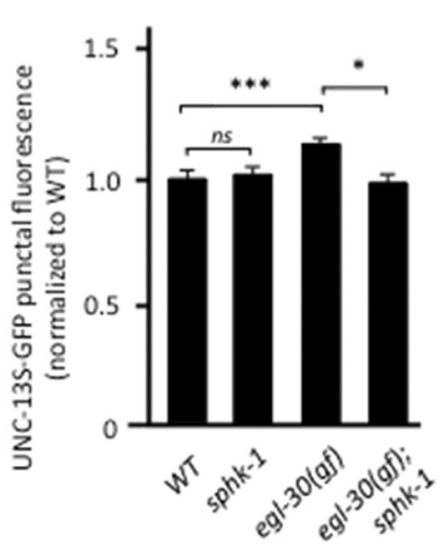

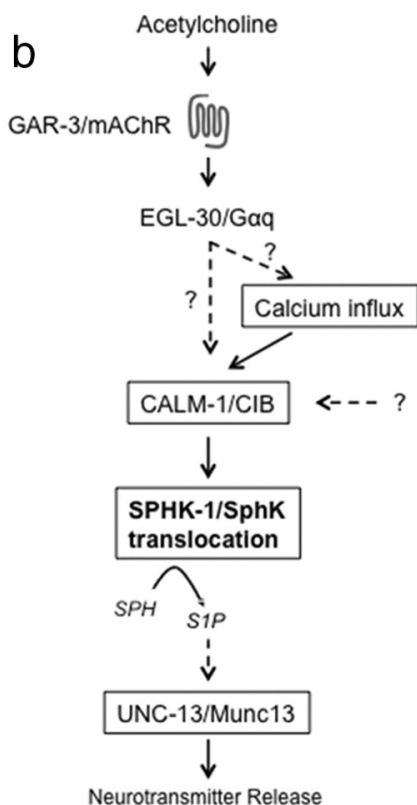

Figure 7. Muscarinic-mediated recruitment of UNC-13/Munc13 requires SPHK-1. $\boldsymbol{a}$, Representative images (left) and quantification (right) of GFP-tagged UNC-13S (UNC-13S-GFP) in axons of $\mathrm{DA} / \mathrm{DB}$ motor neurons analyzed in the indicated strains. ${ }^{*} p<0.05$ and ${ }^{* * *} p<0.0005$, Student's $t$ tests. Scale bar: $10 \mu \mathrm{m} . \boldsymbol{b}$, Model of the muscarinic-and calcium-mediated SPHK-1 translocation for the facilitating effects of muscarinic signaling pathways on neurotransmitter release in C. elegans cholinergic motor neurons. Dotted lines represent unknown pathways or pathways that are likely to be connected via multiple proteins.

muscarinic signaling. We previously showed that SPHK-1 translocation can occur within 15 min in vivo (Chan et al., 2012), but in principal, SPHK-1 synaptic recruitment could occur over much more rapid timescales. A previous study has observed that agonist-dependent translocation of SphK in HEK cells occurs on the order of seconds (ter Braak et al., 2009). Thus, calciumdependent recruitment of SPHK-1 by muscarinic signaling may encode a presynaptic plasticity mechanism during cholinergic activity.

The observation that mutations in any one of three calcium channels reduce SPHK-1-GFP abundance suggests that these channels act nonredundantly to promote SPHK-1 recruitment. Previous studies demonstrate that these channel types can promote calcium entry at presynaptic terminals. UNC-68/RyR is required for proper synaptic release from motor terminals and UNC-68 and mammalian RyRs have been shown to localize at presynaptic terminals (Bouchard et al., 2003; Liu et al., 2005; Nizami et al., 2010). Similarly, EGL-19/L-VGCC contributes to the generation of calcium waves in axons following axotomy (Ghosh-Roy et al., 2010). Finally, UNC-2/VGCC $\alpha 1$ is concentrated at presynaptic active zones and positively regulates release at neuromuscular junctions (Saheki and Bargmann, 2009). However, RyRs also function in cell bodies, where the endoplasmic reticulum is abundant, and EGL-19/L-VGCC $\alpha 1$ promotes calcium influx in neuron cell bodies (Frøkjaer-Jensen et al., 2006). Thus we cannot rule out that these channels may function at a distance (in motor neuron somas) to regulate SPHK-1 recruitment to presynaptic terminals.

Activation of muscarinic receptors drives diverse signaling pathways downstream of $\mathrm{G} \alpha \mathrm{q}$, including those that alter calcium influx into the cytoplasm. In neurons, activation of the M3 subtype of muscarinic receptor classically leads phospholipase $\mathrm{C}$ (PLC)-dependent changes in potassium channel conductance, resulting in changes in neuronal activity (Wess et al., 2007; Brown, 2010). However, muscarinic activation of M3 muscarinic receptors has also been shown to induce calcium influx and to require voltage-sensitive calcium channels and TRP channels (Michel et al., 2005; Tai et al., 2006). In other cell types such as $\beta$-cells, M3 receptor activation leads to PLC-mediated changes in calcium release form internal stores, which drives insulin secretion (Gromada and Hughes, 2006). Thus, calcium influx is an important downstream event stimulated by muscarinic activation and can alter many cellular functions.

How do presynaptic muscarinic signaling and calcium influx converge on synaptic SPHK-1 recruitment? One possibility is that muscarinic signaling directly recruits SPHK-1 to synapses in a calcium-dependent manner. Alternatively, muscarinic signaling might promote calcium influx, which in turn could lead to SPHK-1 recruitment. Our results point to a model whereby muscarinic activation leads to CALM-1/CIB-dependent SPHK-1 recruitment and/or stabilization at synapses (Fig. $7 b$ ). In support of this model, CALM-1/CIB and SPHK-1 colocalized at presynaptic membranes in neurons. In addition, calm-1/CIB was required for normal SPHK-1-GFP levels at synapses and for the muscarinic-dependent recruitment of SPHK-1-GFP to synapses. The calcium/CaM binding domain also was required for SPHK-1-GFP restriction to discrete membrane domains within presynaptic terminals. The function of CALM-1 in neurons may be analogous to its function in HEK cells, where calcium-dependent binding of CIB to the CaM binding domain of SphK is proposed to promote SphK membrane translocation and increased S1P production (Jarman et al., 2010). Interestingly, mammalian CIB1-positive cells are decreased in cortical regions of brains of Alzheimer's disease patients and CIB1 is thought to bind presenilin 2, suggesting that CIB proteins may play a role in the neurodegeneration associated with Alzheimer's disease (Stabler et al., 1999; Bernstein et al., 2005). Given that SphK and S1P levels are also lower in brains of Alzheimer's disease patients (He et al., 2010; Piccinini et al., 2010), it is intriguing to speculate that CIB and SphK may be coordinately misregulated during disease states. 


\section{Sphingolipid enzymes and presynaptic function}

The generation of S1P by SphK is the last step in an enzymatic cascade that converts sphingomyelin (SM) to S1P. Spatial and temporal regulation of the enzymes in this cascade are likely to be critical in precisely regulating the levels and cellular distribution of S1P. We found that at least two sphingolipid enzymes, in addition to SPHK-1, are localized to presynaptic terminals: HYL-1/ CER synthase and TAG-38/S1P lyase. HYL-1/CER synthase appears to be associated with SV pools whereas SPHK-1 and TAG-38 appear to be associated together on the presynaptic plasma membrane near release sites. It is possible that HYL-1/ CER synthase may associate with mitochondria at presynaptic terminals, since it localizes to mitochondria in other cell types (Yu et al., 2007; Deng et al., 2008). The mechanism by which synaptic TAG-38/S1P lyase is regulated appears to be distinct from that of SPHK-1 because SPHK-1-GFP, but not TAG-38GFP, abundance is altered by muscarinic signaling. We speculate that the balance of sphingolipids at synapses is dynamically regulated by the convergence of multiple pathways that individually regulate different enzymatic components both spatially and temporally at presynaptic terminals.

HYL-1/CER synthase drives the sphingolipid cascade toward more SM and less S1P production, whereas SphK drives this cascade toward S1P production. Accordingly, hyl-1/CER synthase mutants have locomotion and aldicarb phenotypes that are opposite to those of sphk-1 mutants. The effects of hyl-1 mutants on neuromuscular junction function may be due to increased S1P levels, which would explain why hyl-1 aldicarb hypersensitivity is blocked by sphk-1 mutations. However, the presence of TAG-38/ $\mathrm{S} 1 \mathrm{P}$ lyase at synapses raises the possibility that $\mathrm{S} 1 \mathrm{P}$ may be rapidly degraded, and that perhaps the degradation products of S1P may impact synaptic transmission.

\section{SPHK-1 is required for G-protein-dependent recruitment of UNC-13/Munc13 in neurons}

One cellular mechanism by which G-protein pathways regulate neuronal activity is through the recruitment of specific synaptic proteins to release sites. UNC-13/Munc13 is a critical priming protein that promotes synaptic vesicle maturation before exocytosis (Lackner et al., 1999; Richmond et al., 1999; Brose et al., 2000; Madison et al., 2005). We found that UNC-13S-GFP, but not the active zone protein UNC-10/RIM1-GFP, is recruited to release sites by constitutively activate egl-30/G $\alpha q$ mutations (Ch'ng et al., 2008), and this recruitment was dependent on sphk-1. However, sphk-1 mutations did not change UNC-13SGFP puncta fluorescence under normal conditions, suggesting that SPHK-1 activity may specifically recruit UNC-13S during situations where neuronal activity is high (e.g., muscarinic/Gprotein activity). This result is consistent with the observation that sphk-1 mutants do not have defects in spontaneous SV release but only exhibit defects in neurotransmitter release in response to evoked stimuli (Chan et al., 2012). How might SPHK-1 recruit UNC-13/Munc13 to synapses? UNC-13/Munc13 is recruited to release sites by DAG binding to its $\mathrm{C} 1$ domain (Lou et al., 2008). SphK has been reported to activate phospholipase D (PLD), which catalyzes the production of the DAG precursor phosphatidic acid (Pasquar é et al., 2011). Thus, local regulation of DAG by a SphK-PLD pathway at synapses might control UNC-13 synaptic recruitment. However, SphK may regulate UNC-13S abundance by other mechanisms since UNC-13/ Munc13 synaptic abundance is also regulated by calcium/CaM, the calcium binding protein Doc2, and by ubiquitination
(Mochida et al., 1998; Aravamudan et al., 1999; Zikich et al., 2008).

We propose that SPHK-1 at synapses may promote neurotransmitter release by two mechanisms. First, when muscarinic signaling is not activated, SPHK-1 may promote release independently of UNC-13/Munc13. Second, under conditions of increased activity (e.g., during muscarinic stimulation) SPHK-1 activity recruits UNC-13/Munc13 to release sites, where it can prime synaptic vesicle for release. Identifying the synaptic targets of S1P will help to elucidate the molecular mechanisms by which SPHK-1 acts.

\section{References}

Aravamudan B, Fergestad T, Davis WS, Rodesch CK, Broadie K (1999) Drosophila UNC-13 is essential for synaptic transmission. Nat Neurosci 2:965-971. CrossRef Medline

Arellano-Carbajal F, Briseño-Roa L, Couto A, Cheung BH, Labouesse M, de Bono M (2011) Macoilin, a conserved nervous system-specific ER membrane protein that regulates neuronal excitability. PLoS Genet 7:e1001341. CrossRef Medline

Bernstein HG, Blazejczyk M, Rudka T, Gundelfinger ED, Dobrowolny H, Bogerts B, Kreutz MR, Kuznicki J, Wojda U (2005) The Alzheimer disease-related calcium-binding protein Calmyrin is present in human forebrain with an altered distribution in Alzheimer's as compared to normal ageing brains. Neuropathol Appl Neurobiol 31:314-324. CrossRef Medline

Bouchard R, Pattarini R, Geiger JD (2003) Presence and functional significance of presynaptic ryanodine receptors. Prog Neurobiol 69:391-418. CrossRef Medline

Brose N, Rosenmund C, Rettig J (2000) Regulation of transmitter release by Unc-13 and its homologues. Curr Opin Neurobiol 10:303-311. CrossRef Medline

Brown DA (2010) Muscarinic acetylcholine receptors (mAChRs) in the nervous system: some functions and mechanisms. J Mol Neurosci 41:340346. CrossRef Medline

Catterall WA, Few AP (2008) Calcium channel regulation and presynaptic plasticity. Neuron 59:882-901. CrossRef Medline

Chan JP, Hu Z, Sieburth D (2012) Recruitment of sphingosine kinase to presynaptic terminals by a conserved muscarinic signaling pathway promotes neurotransmitter release. Genes Dev 26:1070-1085. CrossRef Medline

Ch'ng Q, Sieburth D, Kaplan JM (2008) Profiling synaptic proteins identifies regulators of insulin secretion and lifespan. PLoS Genet 4:e1000283. CrossRef Medline

Darios F, Wasser C, Shakirzyanova A, Giniatullin A, Goodman K, MunozBravo JL, Raingo J, Jorgacevski J, Kreft M, Zorec R, Rosa JM, Gandia L, Gutiérrez LM, Binz T, Giniatullin R, Kavalali ET, Davletov B (2009) Sphingosine facilitates SNARE complex assembly and activates synaptic vesicle exocytosis. Neuron 62:683-694. CrossRef Medline

Deng X, Yin X, Allan R, Lu DD, Maurer CW, Haimovitz-Friedman A, Fuks Z, Shaham S, Kolesnick R (2008) Ceramide biogenesis is required for radiation-induced apoptosis in the germ line of C. elegans. Science 322: 110-115. CrossRef Medline

Dittman JS, Kaplan JM (2006) Factors regulating the abundance and localization of synaptobrevin in the plasma membrane. Proc Natl Acad Sci U S A 103:11399-11404. CrossRef Medline

Earls LR, Hacker ML, Watson JD, Miller DM 3rd (2010) Coenzyme Q protects Caenorhabditis elegans GABA neurons from calcium-dependent degeneration. Proc Natl Acad Sci U S A 107:14460-14465. CrossRef Medline

Fox RM, Von Stetina SE, Barlow SJ, Shaffer C, Olszewski KL, Moore JH, Dupuy D, Vidal M, Miller DM 3rd (2005) A gene expression fingerprint of C. elegans embryonic motor neurons. BMC Genomics 6:42. CrossRef Medline

Frøkjaer-Jensen C, Kindt KS, Kerr RA, Suzuki H, Melnik-Martinez K, Gerstbreih B, Driscol M, Schafer WR (2006) Effects of voltage-gated calcium channel subunit genes on calcium influx in cultured C. elegans mechanosensory neurons. J Neurobiol 66:1125-1139. CrossRef Medline

Frost RJ, Olson EN (2010) Separating the good and evil of cardiac growth by CIB1 and calcineurin. Cell Metab 12:205-206. CrossRef Medline

Ghosh-Roy A, Wu Z, Goncharov A, Jin Y, Chisholm AD (2010) Calcium 
and cyclic AMP promote axonal regeneration in Caenorhabditis elegans and require DLK-1 kinase. J Neurosci 30:3175-3183. CrossRef Medline

Gromada J, Hughes TE (2006) Ringing the dinner bell for insulin: muscarinic $\mathrm{M} 3$ receptor activity in the control of pancreatic beta cell function. Cell Metab 3:390-392. CrossRef Medline

He X, Huang Y, Li B, Gong CX, Schuchman EH (2010) Deregulation of sphingolipid metabolism in Alzheimer's disease. Neurobiol Aging 31: 398-408. CrossRef Medline

Hengst JA, Guilford JM, Conroy EJ, Wang X, Yun JK (2010) Enhancement of sphingosine kinase 1 catalytic activity by deletion of 21 amino acids from the COOH-terminus. Arch Biochem Biophys 494:23-31. CrossRef Medline

Hunt-Newbury R, et al. (2007) High-throughput in vivo analysis of gene expression in Caenorhabditis elegans. PLoS Biol 5:e237. CrossRef Medline

Jarman KE, Moretti PA, Zebol JR, Pitson SM (2010) Translocation of sphingosine kinase 1 to the plasma membrane is mediated by calcium- and integrin-binding protein 1. J Biol Chem 285:483-492. CrossRef Medline

Kajimoto T, Okada T, Yu H, Goparaju SK, Jahangeer S, Nakamura S (2007) Involvement of sphingosine-1-phosphate in glutamate secretion in hippocampal neurons. Mol Cell Biol 27:3429-3440. CrossRef Medline

Kanno T, Nishizaki T, Proia RL, Kajimoto T, Jahangeer S, Okada T, Nakamura S (2010) Regulation of synaptic strength by sphingosine 1-phosphate in the hippocampus. Neuroscience 171:973-980. CrossRef Medline

Kauselmann G, Weiler M, Wulff P, Jessberger S, Konietzko U, Scafidi J, Staubli U, Bereiter-Hahn J, Strebhardt K, Kuhl D (1999) The polo-like protein kinases Fnk and Snk associate with a $\mathrm{Ca}(2+)$ - and integrin-binding protein and are regulated dynamically with synaptic plasticity. EMBO J 18:5528-5539. CrossRef Medline

Kerr R, Lev-Ram V, Baird G, Vincent P, Tsien RY, Schafer WR (2000) Optical imaging of calcium transients in neurons and pharyngeal muscle of C. elegans. Neuron 26:583-594. CrossRef Medline

Lackner MR, Nurrish SJ, Kaplan JM (1999) Facilitation of synaptic transmission by EGL-30 Gqalpha and EGL-8 PLCbeta: DAG binding to UNC-13 is required to stimulate acetylcholine release. Neuron 24:335346. CrossRef Medline

Leisner TM, Liu M, Jaffer ZM, Chernoff J, Parise LV (2005) Essential role of CIB1 in regulating PAK1 activation and cell migration. J Cell Biol 170: 465-476. CrossRef Medline

Liu Q, Chen B, Yankova M, Morest DK, Maryon E, Hand AR, Nonet ML, Wang ZW (2005) Presynaptic ryanodine receptors are required for normal quantal size at the Caenorhabditis elegans neuromuscular junction. J Neurosci 25:6745-6754. CrossRef Medline

Lou X, Korogod N, Brose N, Schneggenburger R (2008) Phorbol esters modulate spontaneous and $\mathrm{Ca} 2+$-evoked transmitter release via acting on both Munc13 and protein kinase C. J Neurosci 28:8257-8267. CrossRef Medline

Madison JM, Nurrish S, Kaplan JM (2005) UNC-13 interaction with syntaxin is required for synaptic transmission. Curr Biol 15:2236-2242. CrossRef Medline

Mahoney TR, Luo S, Nonet ML (2006) Analysis of synaptic transmission in Caenorhabditis elegans using an aldicarb-sensitivity assay. Nat Protoc 1:1772-1777. CrossRef Medline

Mathews EA, García E, Santi CM, Mullen GP, Thacker C, Moerman DG, Snutch TP (2003) Critical residues of the Caenorhabditis elegans unc-2 voltage-gated calcium channel that affect behavioral and physiological properties. J Neurosci 23:6537-6545. Medline

Mello CC, Kramer JM, Stinchcomb D, Ambros V (1991) Efficient gene transfer in C.elegans: extrachromosomal maintenance and integration of transforming sequences. EMBO J 10:3959-3970. Medline

Menuz V, Howell KS, Gentina S, Epstein S, Riezman I, Fornallaz-Mulhauser M, Hengartner MO, Gomez M, Riezman H, Martinou JC (2009) Protection of C. elegans from anoxia by HYL-2 ceramide synthase. Science 324:381-384. CrossRef Medline

Michel FJ, Fortin GD, Martel P, Yeomans J, Trudeau LE (2005) M3-like muscarinic receptors mediate $\mathrm{Ca} 2+$ influx in rat mesencephalic GABAergic neurones through a protein kinase $\mathrm{C}$-dependent mechanism. Neuropharmacology 48:796-809. CrossRef Medline

Mochida S, Orita S, Sakaguchi G, Sasaki T, Takai Y (1998) Role of the Doc2 alpha-Munc13-1 interaction in the neurotransmitter release process. Proc Natl Acad Sci U S A 95:11418-11422. CrossRef Medline
Nizami S, Lee VW, Davies J, Long P, Jovanovic JN, Sihra TS (2010) Presynaptic roles of intracellular $\mathrm{Ca}(2+)$ stores in signalling and exocytosis. Biochem Soc Trans 38:529-535. CrossRef Medline

O'Halloran DM, Altshuler-Keylin S, Lee JI, L'Etoile ND (2009) Regulators of AWC-mediated olfactory plasticity in Caenorhabditis elegans. PLoS Genet 5:e1000761. CrossRef Medline

Okada T, Kajimoto T, Jahangeer S, Nakamura S (2009) Sphingosine kinase/ sphingosine 1-phosphate signalling in central nervous system. Cell Signal 21:7-13. CrossRef Medline

Pasquar é SJ, Gaveglio VL, Giusto NM (2011) Regulation of phosphatidic Acid metabolism by sphingolipids in the central nervous system. J Lipids 2011:342576. Medline

Piccinini M, Scandroglio F, Prioni S, Buccinn á B, Loberto N, Aureli M, Chigorno V, Lupino E, DeMarco G, Lomartire A, Rinaudo MT, Sonnino S, Prinetti A (2010) Deregulated sphingolipid metabolism and membrane organization in neurodegenerative disorders. Mol Neurobiol 41: 314-340. CrossRef Medline

Pitson SM (2011) Regulation of sphingosine kinase and sphingolipid signaling. Trends Biochem Sci 36:97-107. CrossRef Medline

Richmond JE, Jorgensen EM (1999) One GABA and two acetylcholine receptors function at the C. elegans neuromuscular junction. Nat Neurosci 2:791-797. CrossRef Medline

Richmond JE, Davis WS, Jorgensen EM (1999) UNC-13 is required for synaptic vesicle fusion in C. elegans. Nat Neurosci 2:959-964. CrossRef Medline

Richmond JE, Weimer RM, Jorgensen EM (2001) An open form of syntaxin bypasses the requirement for UNC-13 in vesicle priming. Nature 412: 338-341. CrossRef Medline

Saheki Y, Bargmann CI (2009) Presynaptic CaV2 calcium channel traffic requires CALF-1 and the alpha(2)delta subunit UNC-36. Nat Neurosci 12:1257-1265. CrossRef Medline

Siow DL, Anderson CD, Berdyshev EV, Skobeleva A, Natarajan V, Pitson SM, Wattenberg BW (2011) Sphingosine kinase localization in the control of sphingolipid metabolism. Adv Enzyme Regul 51:229-244. CrossRef Medline

Stabler SM, Ostrowski LL, Janicki SM, Monteiro MJ (1999) A myristoylated calcium-binding protein that preferentially interacts with the Alzheimer's disease presenilin 2 protein. J Cell Biol 145:1277-1292. CrossRef Medline

Sumakovic M, Hegermann J, Luo L, Husson SJ, Schwarze K, Olendrowitz C, Schoofs L, Richmond J, Eimer S (2009) UNC-108/RAB-2 and its effector RIC-19 are involved in dense core vesicle maturation in Caenorhabditis elegans. J Cell Biol 186:897-914. CrossRef Medline

Tai C, Kuzmiski JB, MacVicar BA (2006) Muscarinic enhancement of R-type calcium currents in hippocampal CA1 pyramidal neurons. J Neurosci 26:6249-6258. CrossRef Medline

ter Braak M, Danneberg K, Lichte K, Liphardt K, Ktistakis NT, Pitson SM, Hla T, Jakobs KH, Meyer zu Heringdorf D (2009) Galpha(q)-mediated plasma membrane translocation of sphingosine kinase-1 and crossactivation of S1P receptors. Biochim Biophys Acta 1791:357-370. CrossRef Medline

Vashlishan AB, Madison JM, Dybbs M, Bai J, Sieburth D, Ch'ng Q, Tavazoie M, Kaplan JM (2008) An RNAi screen identifies genes that regulate GABA synapses. Neuron 58:346-361. CrossRef Medline

Wess J, Eglen RM, Gautam D (2007) Muscarinic acetylcholine receptors: mutant mice provide new insights for drug development. Nat Rev Drug Discov 6:721-733. CrossRef Medline

Xu K, Tavernarakis N, Driscoll M (2001) Necrotic cell death in C. elegans requires the function of calreticulin and regulators of $\mathrm{Ca}(2+)$ release from the endoplasmic reticulum. Neuron 31:957-971. CrossRef Medline

Yoon KW, Cho JH, Lee JK, Kang YH, Chae JS, Kim YM, Kim J, Kim EK, Kim SE, Baik JH, Naik UP, Cho SG, Choi EJ (2009) CIB1 functions as a Ca(2+)-sensitive modulator of stress-induced signaling by targeting ASK1. Proc Natl Acad Sci U S A 106:17389-17394. CrossRef Medline

Young KW, Willets JM, Parkinson MJ, Bartlett P, Spiegel S, Nahorski SR, Challiss RA (2003) Ca2+/calmodulin-dependent translocation of sphingosine kinase: role in plasma membrane relocation but not activation. Cell Calcium 33:119-128. CrossRef Medline

Yu J, Novgorodov SA, Chudakova D, Zhu H, Bielawska A, Bielawski J, Obeid LM, Kindy MS, Gudz TI (2007) JNK3 signaling pathway activates cer- 
amide synthase leading to mitochondrial dysfunction. J Biol Chem 282: 25940-25949. CrossRef Medline

Zikich D, Mezer A, Varoqueaux F, Sheinin A, Junge HJ, Nachliel E, Melamed
R, Brose N, Gutman M, Ashery U (2008) Vesicle priming and recruitment by ubMunc13-2 are differentially regulated by calcium and calmodulin. J Neurosci 28:1949-1960. CrossRef Medline 